\title{
Semi-diurnal pressure fluctuation in the ERA40 Data
}

\author{
KLAUS P. HOINKA* \\ Institut für Physik der Atmosphäre, DLR, Oberpfaffenhofen, Germany \\ (Manuscript received December 11, 2006; in revised form May 5, 2007; accepted May 11, 2007)
}

\begin{abstract}
In a regional analysis the removal of the globally present semi-diurnal pressure signal could be necessary in order to isolate the pressure signal of regional features. As soon as global reanalysis data is used an essential prerequisite is to determine the characteristic signal of this data. The semi-diurnal surface pressure fluctuations between 1958 and 2001, deduced from the ERA40 reanalysis data provided by the European Centre for Medium Range Weather Forecasts, are approximatively determined. Annual mean global structure and magnitude of the ERA40 related semi-diurnal pressure tide compare reasonably well with the structure derived from theory and other reanalysis data. However, the comparison with observed data shows regional shortcomings due to the low temporal resolution of the six-hourly ERA40 data. Along the equator the annually mean amplitude of the semi-diurnal pressure fluctuation in the ERA40 data amounts to $1.34 \mathrm{hPa}$. The seasonal values are $1.23 \mathrm{hPa}$ for northern hemispheric summer and $1.39 \mathrm{hPa}$ for winter.
\end{abstract}

\begin{abstract}
Zusammenfassung
Die halbtägig global umlaufende Druckwelle muss oft bei regionalen Analysen herausgefiltert werden, um das mit der regionalen Zirkulation verbundene, isolierte Bodendrucksignal zu erhalten. Werden globale Reanalyse Daten herangezogen, so ist es notwendig, das für diese Daten charakteristische Signal zu bestimmen. Die Amplitude der Halbtageswelle des Bodendruckes wird für die ERA40 Reanalyse Daten des European Centre for Medium Range Weather Forecasts der Periode 1958-2001 annäherungsweise bestimmt. Die globalen Jahresmittel von Struktur und Betrag der für die ERA40 Daten charakteristischen Halbtageswelle sind vergleichbar jener, wie sie sich aus Theorie und anderen Reanalyse Daten ergeben haben. Der Vergleich mit Beobachtungen zeigt jedoch regionale Mängel. Grund dafür ist die geringe zeitliche Auflösung der sechsstündigen ERA40 Daten. Für diese Daten ergibt sich entlang des Äquators eine Amplitude der mittleren jährlichen halbtägigen Bodendruckschwankungen von $1.34 \mathrm{hPa}$. Die saisonalen Werte betragen $1.23 \mathrm{hPa}$ für den nordhemisphärischen Sommer und $1.39 \mathrm{hPa}$ für den entsprechenden Winter.
\end{abstract}

\section{Introduction}

Most regional atmospheric phenomena undergo a diurnal variation with weak to moderate surface pressure fluctuations, such as land- and sea-breezes, mountainvalley winds and thermal lows. For instance, in Iberian thermal lows the amplitude of the diurnally varying pressure amounts to about $1 \mathrm{hPa}$ (HOINKA and CASTRO, 2003). This weak signal might be masked by the globally acting semi-diurnal pressure fluctuation due to various reasons e.g. among others the stratospheric ozone heating. In order to isolate the pressure signal of the related atmospheric phenomenon it is necessary to remove locally the globally present semi-diurnal signal. Surface observations as well as numerical simulations provide data with sufficient temporal resolution of at least one hour which allows to analyse the semi-diurnal pressure wave locally and to remove it from the pressure field. However, in the case of regional analyses based on global reanalysis data this is not possible, because their

${ }^{*}$ Corresponding author: Klaus P. Hoinka, Institut für Physik der Atmosphäre, DLR, Postfach 1116, 82230 Wessling, Germany, e-mail: klaus.hoinka@dlr.de six hourly resolution is too coarse to determine the local semi-diurnal pressure oscillation.

Based on global analysis of surface station and marine data, recently DAI and WANG (1999) have shown the complicate global picture of the surface pressure anomalies. Unfortunately, the global structures determined by DAI and WANG (1999) cannot be used for removing the semi-diurnal pressure cycle from regional ERA40 analyses because structure and amplitude depend on the used data. These amplitudes and the structure might not be adequate being applied for a different data set, e.g. ERA40 data. DAI and WANG (1999) pointed out that the predictions of HAURWITZ (1956) tidal theory have some inaccuracies due to limited data which he used in his studies. Nevertheless, the predictions of tidal theory based on HAURWITZ (1956) is a globally useful, reasonable and simple appproach for giving a rough estimate of the semi-diurnal tidal wave. Based on an empirical calculation, HAURwITZ (1956) showed that the zonal semi-diurnal travelling pressure wave $\left(p_{2}\right)$ can be expressed as

$$
p_{2}=A_{2} \times \cos ^{3}(\phi) \times \sin \left[2 \times\left(t^{\prime}+\lambda\right)+158^{\circ}\right]
$$

where $\mathrm{p}$ is the surface pressure in $\mathrm{hPa}, \phi$ is latitude, $\lambda$ 
is east longitude, and $t^{\prime}$ is UTC reckoned in angle at the rate of $360^{\circ}$ per mean solar day from the lower transit (i.e., midnight). The equation indicates that the semidiurnal pressure $p_{2}$ has its maximum in the Tropics and decreases poleward. The maximum occurs around 10 a.m. and p.m. local time. The tides are travelling at a speed of roughly $90^{\circ}$ of longitude westward per 6 hours. The amplitude $A_{2}$ (in $\mathrm{hPa}$ ) is the key parameter because its value determines the amplitude of the local pressure signal $p_{2}$. Based on observational data, numerical simulations and reanalysis data, the amplitude $A_{2}$ results in different magnitudes ranging from about 1.0 to $1.5 \mathrm{hPa}$ (DAI and WANG, 1999).

The work of HSU and Hoskins (1989) suggests that tidal fluctuations in the troposphere are reasonably well preserved in the model of the European Centre for Medium Range Weather Forecasts (ECMWF). The use of this type of model data implies a model-inherent presentation of the tides. This is due to the fact that results obtained from gridded model data may be sensitive to the details of the representation of the stratosphere in the model because the semi-diuirnal cycle is excited e.g. by ozone heating in the stratosphere and mesosphere. The ECMWF model was used to generate time series of reanalysed data, ERA15 and ERA40. The recently available ERA40 are widely used to study not only global but also regional phenomena where it might be necessary to remove the semi-diurnal pressure tide. However, a study of tidal characteristics of the ERA40 reanalysis data has not been done so far. Therefore, in this study the amplitude of the semi-diurnal pressure fluctuations $A_{2}$ is determined which is characteristic for the ERA40 data series. For a rough estimate this amplitude can then be used to remove $p_{2}$ from regionally analysed data based on ERA40 by applying the equation of HAURWITZ (1956). However, one has to keep in mind that the study of the general tidal characteristics of this reanalysis data series is outside of the scope of this paper.

\section{Data and method}

The data used are the ERA40 reanalysis (UPPALA et al., 2005) at a spectral truncation T157 and the ERA15 reanalysis at T106 (GIBSON et al., 1997). The surface pressure fields are interpolated to a $5^{\circ}$ grid (in lat and lon) and are used from 1958 through 2001, sampled four times a day. For each observing time, 00 UTC for instance, and at each grid point annual and seasonal means were obtained by averaging the daily fields over the period 1958-2001. In a second step the grid point daily means were removed from the six-hourly climatological fields. The resulting residual fields were then harmonically analysed to obtain the amplitude of the semidiurnal cycle. All the four times (00, 06, 12 and 18 UTC) are treated independently from each other.
Following VAN DEN DoOL et al. (1997), the residual fields are interpolated in time and global semi-diurnal pressure states are obtained at an hourly resolution. Normally the phase speed between various times is not constant as has been shown by VAN DEN DOOL et al. (1997). For simplicity, in this study the phase speed is kept constant during the entire day. In applying the wave interpolation procedure the series of interpolated fields pass exactly through the observed states at 00, 06, 12 and 18 UTC and vary smoothly in between. Finally, at each grid-point the mean amplitude of the semi-diurnal oscillation is determined using the hourly interpolated fields by removing the diurnal cycle as well as periods shorter than 12 hours.

\section{Results}

Figure 1 (four panels of left column) shows the annual mean deviations of the 00, 06, 12 and 18 UTC surface pressure climatologies from the daily mean climatology. The shape of the pressure anomalies is somewhat ragged and outlines details of the continent borders in some places at certain times, e.g. in Latin America at 00/12/18 UTC and Australia at 00/06 UTC. Although the semi-diurnal cycle is known to be dominant, it is obvious that maps separated by 12 hours are not identical, nor are the maps separated by 6 hours the opposite of each other. The structure compares well with those obtained from surface station data (DAI and WANG, 1999) and reanalysis data (VAN DEN Dool et al., 1997). The amplitudes show the same magnitude as those presented by VAN DEN DOOL et al. (1997) but stronger than those obtained from station data (DAI and WANG, 1999), however, the structure during 00, 06, 12 and 18 UTC is about the same (see their Fig.2). The zonal wavenumber two predominates clearly the total fields over low and middle latitudes but becomes less evident in high latitudes. The fields exhibit at all times two maxima and minima where the anomalies are largest over South-America, Africa and Australia.

In Fig.1 (four panels of right column) is given the local appearance of the semi-diurnal wave after removing the diurnal cycle and periods shorter than 12 hours. The four panels exhibit the state of the semi-diurnal oscillation only at four times UTC in order to underline the difference to the original fields. Since the semi-diurnal fluctuations exhibit little seasonal variation, only the climatological mean is shown here. A characteristic feature is that the negative amplitudes are stronger than their positive counterparts at 00 and 12 UTC, whereas the opposite is the case for 06 and 18 UTC. This was already noted by HSU and HOSKINS (1989) and VAN DEN DoOL et al. (1997).

A similar calculation was performed based on a spatial resolution of $1.125^{\circ}$. The aim was to test if local effects, e.g. orography, have an impact on the semi-diurnal 

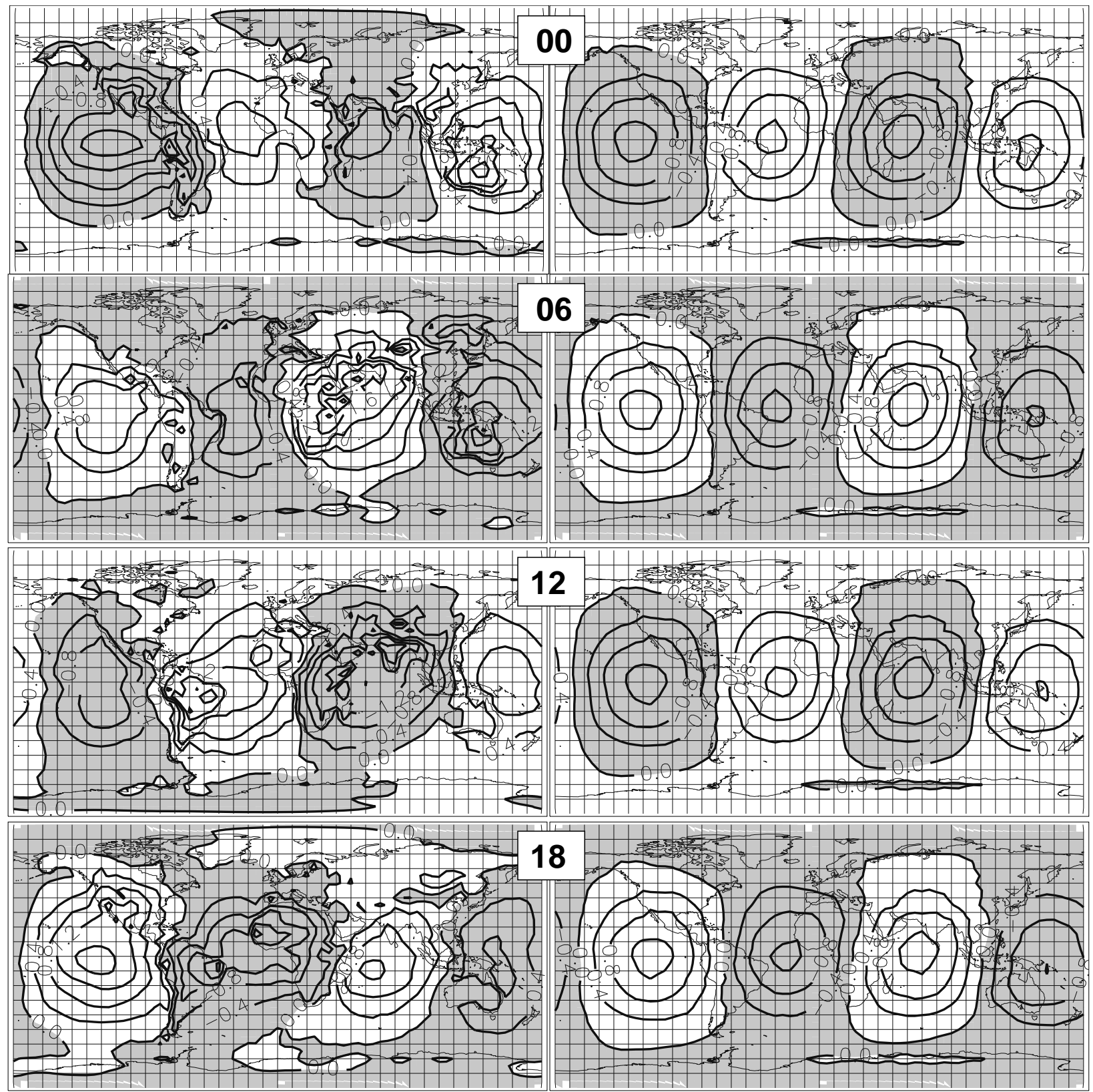

Figure 1: Climatological mean surface pressure anomaly (left column) and the pure semi-diurnal pressure oscillation (right column) at 00, 06, 12 and 18 UTC averaged over the period 1958-2001. Anomaly is defined as departure of the climatology at a given time from the daily mean. Increment is $0.4 \mathrm{hPa}$; negative values are shaded.

tidal wave. This is the best grid point resolution of the ERA40 data corresponding to the resolution in spectral space. At a higher resolution the grid point values are simply interpolated. The results show that the structure given in Fig.1 (left column) in amplitudes and phase is about the same, except that the smooth isolines exhibit a more ragged character. In particular, this is valid in locations of significant orography of the Andes and the Tibetian Plateau at 00 and 12 UTC. The amplitude of the semi-diurnal fluctuation increases by about $2 \%$ as soon as the grid size is decreased to $1.125^{\circ}$. The fact that orography does not have an impact on the semi-diurnal tidal wave is already shown by DAVIES and PHILLIPS
(1985) who found a symmetric behaviour of amplitude and phase of the semi-diurnal tidal wave in a north-south cross section across the Alps. In contrast to this, significant differences were encountered in the diurnal tidal pressure fields between north and south of the Alps.

Figure 2 shows the global picture of the local amplitudes of the semi-diurnal oscillation for the annual mean (a), the deviations of northern hemispheric summer mean from the annual mean (b) and the corresponding winter deviations (c). The annual averaged amplitude $p_{2}$ is largest $(1.34 \mathrm{hPa})$ in the Tropics over SouthAmerica, the eastern Pacific, Africa and north of Australia. In mid-latitudes the amplitude is uniform zonally. 

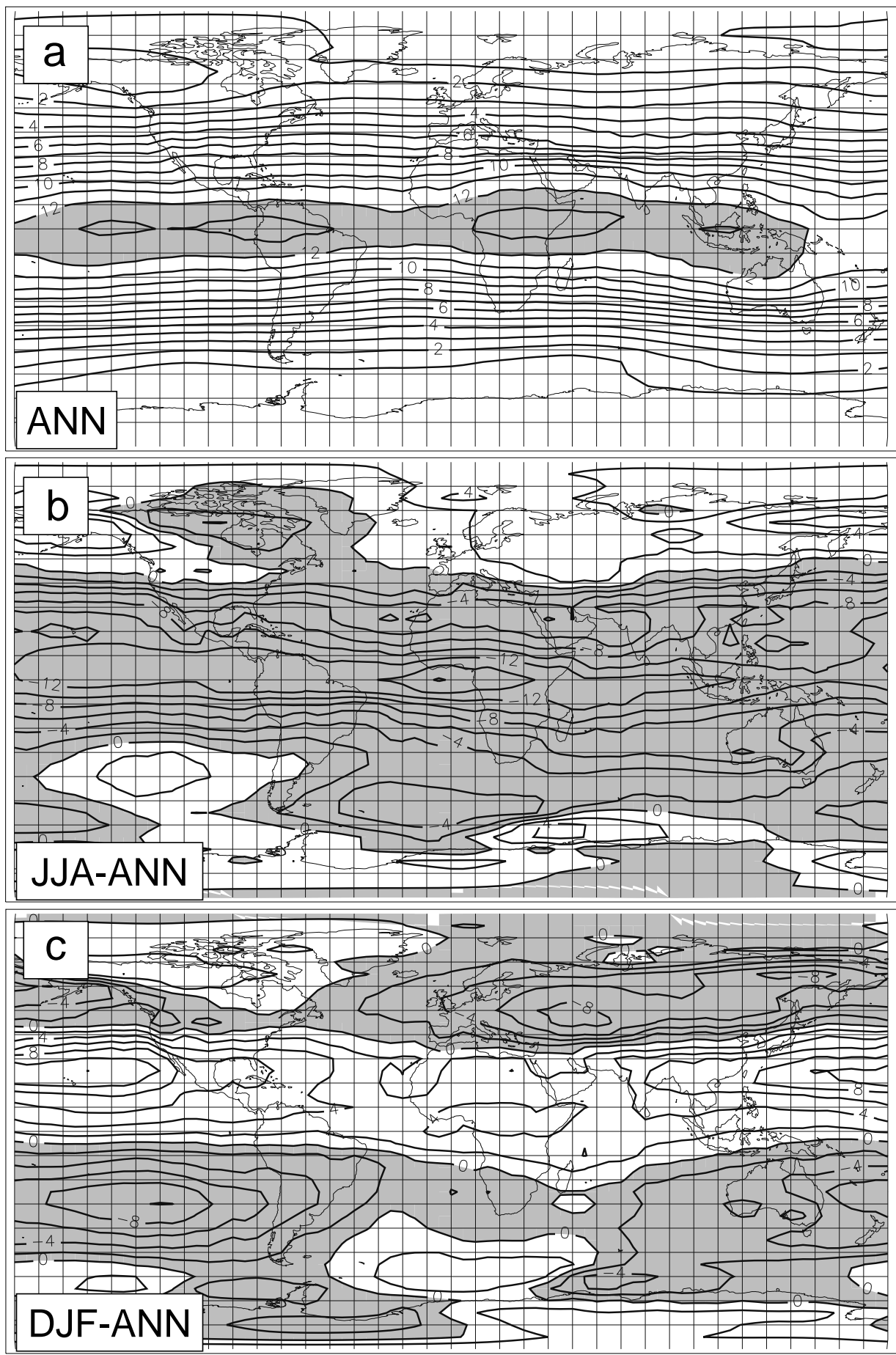

Figure 2: Mean amplitude (in units of $0.1 \mathrm{hPa}$ ) of the ERA40 semi-diurnal oscillation of the surface pressure (a) averaged over the period 1958-2001; deviations of the northern-hemispheric summer mean from the annual mean (b) in $0.01 \mathrm{hPa}$; and the corresponding winter deviations (c) in $0.01 \mathrm{hPa}$. The shading marks regions larger than $1.2 \mathrm{hPa}(\mathrm{a})$ and negative deviations (b, c).

The belt with strongest meridional gradients is located in both hemispheres limited roughly by $15^{\circ}$ and $50^{\circ}$ latitudes. These structures compare nicely with the results from GEOS-1 Reanalysis data (DAI and WANG, 1999; their Fig.16) except that in the latter zonal gradients appear above the northern Atlantic Ocean and southern Latin America.
Compared with station data (DAI and WANG, 1999; their Fig. 8), Fig. 2 reveals as expected less regional and zonal variations, although the amplitude is comparable in magnitude over most of the areas. The amplitude's structure based on station data exhibits a great deal of low resolution features due to the temporal resolution of the data. The amplitude of the semi-diurnal 
Table 1: Amplitude $p_{e q}(\mathrm{hPa})$ of the semi-diurnal tide of the annual-mean surface pressure along the equator from various sources. * Version 1 of the Data Assimilation System of the Goddard Earth Observing System.

\begin{tabular}{lcccc}
\hline source & data & period & type & $p_{e q}$ \\
\hline HAURWITZ (1956) & station data & & theory & 1.16 \\
LINDZEN (1990) & station data & & theory & 1.10 \\
DAI and WANG (1999) & station data & $76-97$ & observation & 1.10 \\
HSU and HOSKINS (1989) & ECMWF model & $86-87$ & simulation & 1.16 \\
WoOLNOUGH et al. (2004) & GCM HadCM3 & 90 days & simulation & 1.15 \\
VAN DEN DoOL et al. (1997) & NCAR/NCEP & $79-95$ & reanalysis & 1.47 \\
DAI and WANG (1999) & GEOS-1* & $80-94$ & reanalysis & 1.41 \\
this study & ECMWF ERA15 & $79-93$ & reanalysis & 1.25 \\
this study & ECMWF ERA40 & $58-01$ & reanalysis & 1.34 \\
\hline
\end{tabular}

tide exhibits considerable regional and zonal variations. See DAI and WANG (1999) for a more detailed discussion of these features. In the station data analysis, additional strong maxima appear over the eastern and western Pacific, and the Indian Ocean. The amplitude in the ERA40 reanalysis is more uniform zonally, about 0.2 $0.3 \mathrm{hPa}$ smaller at high latitudes than observed, particularly above the Atlantic Ocean and the continent of Asia. The observations show to the west of continents a northerly bulging of the isolines and a southerly one at the continent's lee side. This is apparent above Northand South-America, Africa and Europe, East-Asia and Australia. In the ERA40 data there are some indications of this bulging to the west of North- and South-America.

The seasonal means in the amplitude in the Tropics amounts to $1.23 \mathrm{hPa}$ (JJA) and $1.39 \mathrm{hPa}$ (DJF) (not shown). In summer the sub-tropical and tropical amplitude decrease in both hemispheres. The strongest decrease of down to $-0.13 \mathrm{hPa}$ is found close to Africa. A significant decrease appears above the northern Pacific Ocean and a weak one west of Latin America. Above the northern hemispheric sub-tropical and tropical belt the amplitudes increase by more than $0.1 \mathrm{hPa}$, particularly over the tropical Pacific Ocean. The amplitudes decrease above the southern Subtropics and Tropics except above Afrika. The maximum decreases appear above the western South-Pacific and above Asia. All this is in accord with the seasonal variation resulting from surface station data (DAI and WANG, 1999).

The intercomparion of the amplitude's global picture as obtained from station data, GEOS- 1 analyses and ERA40 reanalyses shows that the latter yields a global picture with weak regional and zonal details. The reason for this shortcoming is apparently the low temporal resolution of the ERA40 data. The intermediate times 03, 09, 15, 21 UTC appearing in Fig. 2 of DAI and WANG (1999) cannot be constructed from the ERA40 data. In particular, those times are missing in the ERA40 data which are close to the local passage of the semidiurnal pressure maximum at 10 a.m. and p.m. Thus the ERA40 data show less regional structures. Never- theless, the aim of this study is to get a rough estimate of the amplitude $A_{2}$. In order to compare the determined ERA15/ERA40 amplitudes with those from other sources, Tab. 1 lists the amplitudes $A_{2}$ of the semidiurnal tide of the annual mean surface pressure along the equator. The amplitude is $1.16 \mathrm{hPa}$ as forecasted by tidal theory (HAURWITZ, 1956). More recent calculations based on an increased number of surface station determined $1.10 \mathrm{hPa}$ (LINDZEN, 1990). Based on global surface pressure data, DAI and WANG (1999) determined an amplitude of 1.10 , with seasonal variations between 1.0 and $1.3 \mathrm{hPa}$. Obviously, GCM simulations tend to slightly overestimate the amplitude $A_{2}$ (HSU and Hoskins, 1989; WoOLNOUGH et al., 2004).

Reanalysis (VAN DEN Dool et al., 1997; DAI and WANG, 1999) appears to have stronger tides than what has been reported classically based on hourly surface station data. The magnitudes determined from ERA15 $(1.25 \mathrm{hPa})$ and ERA40 (1.34 hPa) data are obviously in the typical range for reanalysis data. The top layer of the ECMWF model version used for the ERA15 reanalysis is at $10 \mathrm{hPa}$ (near $30 \mathrm{~km}$ ), well below the height of maximum ozone heating $(40-50 \mathrm{~km})$. Accordingly, the forcing for semi-diurnal tides should be underestimated in this model version and the amplitude of the semidiurnal tides in the model is expected to be smaller than the value obtained using ERA40. This model version has its top level at $0.1 \mathrm{hPa}$ (near $65 \mathrm{~km}$ ) thus including the layer of maximum ozone heating.

The amplitudes listed in Tab. 1 vary significantly depending on the used data type. WoOLNOUGH et al. (2004) pointed out that the use of 3-hourly mean diagnostics results in an amplitude reduction of about $10 \%$ for the semi-diurnal harmonics compared to using instantaneous fields. DAI and WANG (1999) stated that the uncertainty is about $10 \%$ if 3-hourly mean diagnostics are used. Due to the lower resolution of the six-hourly ERA40 data it is expected that the uncertainty will increase. An exact calculation of the uncertainty does not make sense here because the resulting amplitudes are used as a rough estimate. 


\section{Concluding remarks}

In the present study the global amplitude characteristic for the semi-diurnal pressure fluctuations is determined for the ERA40 data. The global structure is found to be in general agreement with the results of previous investigators and predictions of tidal theory. Along the equator the amplitudes of the semi-diurnal pressure fluctuation results to $1.25 \mathrm{hPa}$ (ERA15) and 1.34 (ERA40). This suggests that tidal fluctuations at the surface are reasonably well preserved in the ERA40 data. Thus, a simple procedure for removing the semi-diurnal pressure fluctuation from regionally analysed data based on ERA40 is to apply the equation of HAURWITZ (1956) by using these amplitudes for $\mathrm{A}_{2}$.

Clearly 6-hourly reanalysis data is hampered by the coarse temporal resolution. At present it is not clear why reanalysis data have in general too strong tides surmounting amplitudes clasically based on hourly surface station data or classical theory. One reason might be the fact that results obtained from gridded model data are sensitive to the details of the representation of the stratosphere in the model. ZWIERS and HAMILTON (1986) showed that excluding the ozone heating above $10 \mathrm{hPa}$ could lead to a $43 \%$ reduction in amplitude of the semi-diurnal tide. Besides the thermal forcing of the stratospheric ozone, tropospheric water vapor plays also its role in forcing the semi-diurnal oscillation. Sensitive experiments performed by WoOLNOUGH et al. (2004) indicated that stratospheric ozone forcing is less dominant and the water vapor shortwave absorption in the troposphere is more important than previously thought. Thus, the amplitude of the semi-diurnal pressure wave as analysed in reanalysis data or simulated in numerical models might indicate to a certain degree the quality of the implemented stratosphere and tropospheric water vapor cycle.

The pressure amplitude of the semi-diurnal amplitude is small in comparison with synoptic pressure changes and it might be questioned whether the phenomenon has any practical significance. Obviously, one practical significance is to isolate the phenomenonrelated pressure signal. Moreover, the semi-diurnal pressure oscillations are well known by the weather forecasters because the amplitude is relatively large and must be subtracted from pressure tendencies before these can be used in forecasting. This effect is strongest in the Tropics whereas in midlatitudes the pressure fluctuations are particularly notable in homogeneous pressure conditions when the forecast is normally an easy task. The semi-diurnal oscillation might be dynamically active in some occasions. The existence of pressure gradients associated with the travelling semi-diurnal pressure perturbations at the surface and their extension upward into the earth's atmosphere imply that tidal wind oscillations will also occur. WHITEMAN and BIAN (1996) calculated from radar profiler data that the tidal wind velocity is quite weak near the ground but increases with height to attain around $1 \mathrm{~m} / \mathrm{s}$ in the mid troposphere.

\section{References}

DAI, A., J. WANG, 1999: Diurnal and semidiurnal tides in global surface pressure fields. - J. Atmos. Sci. 56, 38743891.

Davies, H.C., P.D. Phillips, 1985: Mountain drag along the Gotthard section during ALPEX. - J. Atmos. Sci. 42, 2093-2109.

GibSON, R., P. KÅllberg, S. UpPala, A. Hernandez, A. Nomura, E. SERrano, 1997: ERA Description. ECMWF-ReAnalysis project Report Series 1, 72 pp.

HAURWITZ, B., 1956: The geographical distribution of the solar semi-diurnal pressure oscillation. - New York Univ. Coll. Engl. Meteor. Pap. 2, 1-36.

Hoinka, K.P., M. CAstro, 2003: The Iberian Peninsula thermal low. - Quart. J. Roy. Meteor. Soc. 129, 1491-1511.

HsU, H., B.J. Hoskins, 1989: Tidal fluctuations as seen in ECMWF data. - Quart. J. Roy. Meteor. Soc. 115, 247-264.

Lindzen, R.S., 1990: Dynamics in Atmospheric Physics. Cambridge University Press, $310 \mathrm{pp}$.

Uppala, S.M., P.W. Kallberg, A.J. Simmons, U. ANDRAE, V. DA COSTA Bechtold, M. Fiorino, J.K. Gibson, J. Haseler, A. Hernandez, G.A. Kelly, X. Li, K. Onogi, S. SAarinen, N. SokKa, R.P. ALlan, E. Andersson, K. Arpe, M.A. Balmaseda, A.C.M. BeljaARs, L. VAN De Berg, J. Bidlot, N. Borman, S. Caires, F. Chevallier, A. Dethof, M. Dragosavac, M. Fisher, M. Fuentes, S. HageMANN, E. Holm, B.J. Hoskins, L. ISAKSEN, P.A.E.M. Janssen, R. JENne, A.P. MCNALly, J.-E. MAhFouf, J.-J. Morcrette, N.A. RAYNer, R.W. SAunders, P. Simon, A. Sterl, K.E. Trenberth, A. Untch, D. VAsiljeVic, P. Viterbo, J. Wollen, 2005: The ERA40 re-analysis. - Quart. J. Roy. Meteor. Soc. 131, 29613012.

VAN DEn DoOl, H.M., S. SAHA, J. SChremM, J. HuANG, 1997: A temporal interpolation method to obtain hourly atmospheric surface pressure tides in Reanalysis 1979-1995. - J. Geophys. Res. 102, 22013-22024.

Whiteman, C.D., X. Bian, 1996: Solar semidiurnal tides in the troposphere: Detection by radar profilers. - Bull. Amer. Meteor. Soc. 77, 529-542.

WoOlnOUgh, S.J., J.M. SLINGO, B. Hoskins, 2004: The diurnal cycle of convection and atmospheric tides in an aquaplanet GCM. - J. Atmos. Sci. 61, 2559-2573.

Zwiers, F., K. Hamilton, 1986: Simulation of solar tides in the Canadian Climate Centre General Circulation Model. - J. Geophys. Res. 91, 11877-11896. 